\title{
A Novel ATP1A2 Mutation in Pediatric Hemiplegic Migraine
}

\author{
Christopher J Butler* and Andrew A Mallick
}

Department of Pediatric Neurology, Bristol Royal Hospital for Children, Bristol, United Kingdom

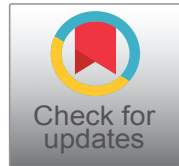

*Corresponding author: Dr. Christopher J Butler, Department of Pediatric Neurology, Bristol Royal Hospital for Children, Bristol, United Kingdom

\begin{abstract}
Background: Migraine is a common pediatric disorder presenting with unilateral frontal headache. Hemiplegic migraine is a rare and clinically challenging subtype of migraine with aura. There are two different subtypes of hemiplegic migraines; they can occur as either sporadic or familial forms. Recently our understanding of the genetics underlying hemiplegic migraine has rapidly evolved. Familial hemiplegic migraine has been associated with pathogenic mutations in three independent genes; CACNA1A, ATP1A2 and SCN1A.
\end{abstract}

Case presentation: An 11-year-old boy presented with a sudden onset severe frontal headache with associated facial asymmetry and limb weakness on the left. A cranial magnetic resonance imaging (MRI) scan and an electroencephalogram (EEG) was performed which were both reported as normal. A familial hemiplegic migraine gene panel revealed a novel heterozygous mutation C.1159 G > A on exon 9 of the ATP1A2 gene.

Conclusion: This case report describes a boy with typical features of hemiplegic migraine and a family history of migraine with aura. Genetic analysis revealed a previously undescribed heterozygous mutation in the ATP1A2 gene.

\section{Keywords}

Hemiplegic migraine, ATP1A2, Migraine, Hemiplegia, Case report

\section{Abbreviations \\ CT: Computed Tomography; EEG: Electroencephalogram ICHD-3: International Classification of Headache Disorders $3^{\text {rd }}$ Edition, Beta Version; MRI: Magnetic Resonance Imag- ing; MRA: Magnetic Resonance Imaging with Angiography}

\section{Background}

Migraine is a common pediatric disorder typically presenting with severe unilateral frontal headache which can be associated with aura in up to a third of patients [1]. Hemiplegic migraine is a rare and clinically challenging subtype of migraine with aura. A key feature is a fully reversible motor aura which typically presents as a hemiparesis. Additional aura types are commonly present which can include disturbances of sensation, vision and speech [2].

The International Classification of Headache Disorders ( $3^{\text {rd }}$ edition), beta version (ICHD-3) classifies hemiplegic migraines as having two separate subtypes; these are either sporadic or familial forms [2]. Familial hemiplegic migraines are diagnosed in patients with at least one first- or second-degree relative with migraine aura, including motor weakness. Sporadic hemiplegic migraine is diagnosed in the absence of such a family history.

There are few epidemiological studies of hemiplegic migraines. However, Danish studies have reported the prevalence of the sporadic form to be 1 in 10,000 people with, the sporadic and familial form equally frequent [3]. The clinical symptoms and signs observed in both familial and sporadic subtypes are comparable. Patients with hemiplegic migraine have reported multiple exacerbating factors such as minor head injuries, exercise and emotional stress [4]. In addition to the typical motor and sensory auras, patients are at risk of basilar-type auras which can present as encephalopathy and coma [1]. Shared symptomatology between hemiplegic migraine and focal seizures has meant that attacks can easily be misdiagnosed as epilepsy [2]. Furthermore, it should be noted that the common mutations found in hemiplegic migraine are independently associated with a higher risk of epilepsy [1].

Recently our understanding of the genetics underlying familial hemiplegic migraine has rapidly evolved.

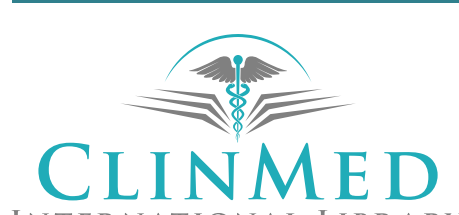

INTERNATIONAL LIBRARY
Citation: Butler CJ, Mallick AA (2018) A Novel ATP1A2 Mutation in Pediatric Hemiplegic Migraine. Int J Pediatr Res 4:043. doi.org/10.23937/2469-5769/1510043

Accepted: October 31, 2018: Published: November 02, 2018

Copyright: (C) 2018 Butler CJ, et al. This is an open-access article distributed under the terms of the Creative Commons Attribution License, which permits unrestricted use, distribution, and reproduction in any medium, provided the original author and source are credited. 
Table 1: Familial subtypes of hemiplegic migraine with associated gene involved. This case report documents a novel genetic mutation in the ATP1A2 gene in a child with hemiplegic migraine.

\begin{tabular}{|l|l|l|l|}
\hline Subtype & Gene & Protein involved & Neuronal ion channel affected \\
\hline Familial hemiplegic migraine type 1 (FHM1) & CACNA1A & Alpha-1 subunit & Voltage-gated Calcium channel \\
\hline Familial hemiplegic migraine type 2 (FHM2) & ATP1A2 & Alpha-2 subunit & Sodium-Potassium ATPase pump \\
\hline Familial hemiplegic migraine type 3 (FHM3) & SCN1A & Alpha-1 subunit & Voltage-gated Sodium channel \\
\hline
\end{tabular}

Familial hemiplegic migraine has been associated with pathogenic mutations in three independent genes; CACNA1A, ATP1A2 and SCN1A [1]. These genes encode three separate ion channels. Each ion channel involved has a heterogeneous migraine phenotype which can lead to three subtypes of familial hemiplegic migraine [1] (Table 1).

\section{Case Presentation}

An 11-year-old boy presented to our institution after suffering from a sudden onset and severe headache whilst playing football at school. Additionally, he described numbness in his left hand and left cheek. A cranial computed tomography (CT) and magnetic resonance imaging (MRI) with angiography was reported as normal. The numbness resolved over a few days and he was discharged with outpatient follow up.

Two weeks later he complained of a sudden onset frontal headache with photophobia, blurred vision and nausea. His parents described facial asymmetry with right sided drooping and marked dysarthria. During the episode it was noted that he had right sided upper and lower limb motor weakness with altered sensation.

Over the next 8 months he had a further 5 episodes of rapid onset headache, nausea, photophobia and unilateral limb and facial weakness. He was readmitted eight months after the initial presentation due to a severe frontal headache with photophobia and a temperature of $100.8^{\circ} \mathrm{F}$. On examination he had a left sided facial weakness with reduced power in his left upper and lower limbs. Due to the nature of the presenting features plus a fever the main differential diagnosis was meningoencephalitis; therefore, intravenous antibiotics (Ceftriaxone, $80 \mathrm{mg} / \mathrm{kg}$ ) were initiated. Additionally, Sumatriptan (50 mg, Tablet) was given in the emergency department with a positive effect. A cranial MRI with angiography (MRA) was repeated and reported by local pediatric neuroradiologists as normal. An electroencephalogram (EEG) at the time of his headache was also reported by local neurophysiologists as being within normal range. His symptoms resolved over a 24-hour period.

Prior to the first presentation to our institution the patient had a 2-year history of migraine without aura which occurred at a frequency of two per year. He had not experienced hemiplegia before age 11 years. Both his mother and maternal grandmother have migraine with typical aura (visual) but neither have a history of hemiplegia.
A familial hemiplegic migraine next generation sequencing gene panel was sent to Sheffield Diagnostic Genetic Service, UK which revealed a novel heterozygous mutation c.1159 $\mathrm{G}>\mathrm{A}$ on exon 9 of the ATP1A2 gene.

\section{Discussion}

This case report describes a boy with typical features of hemiplegic migraine and a family history of migraine with typical aura. Genetic analysis revealed a previously undescribed heterozygous c. (1159 G > A) mutation of unknown significance in the $A T P 1 A 2$ gene.

The ATP1A2 gene encodes the alpha- 2 subunit of neuronal sodium-potassium ATPase pumps. Our patient's novel mutation is predicted to result in an Alanine to Threonine amino acid substitution at residue 387 in a conserved functional domain of the alpha-2 subunit protein. In silico evolutionary conservation analysis on this mutation supports a likely deleterious effect on the alpha-2 subunit.

Sodium-Potassium ATPase pumps are responsible for maintaining electrochemical gradients across cellular plasma membranes. This gradient is essential for the excitability of neurons, cellular uptake of organic and inorganic molecules and osmoregulation [5]. Altered function of the mutated alpha- 2 subunits have been hypothesised to reduce neuronal reuptake of potassium and glutamate leading to slow recovery following neuronal excitation. This culminates in increased concentrations of potassium and glutamate in the synaptic cleft which has been shown to cause cortical spreading depression with prolonged neuronal inhibition and activation of the trigeminovascular system $[1,5]$.

\section{Conclusion}

This case illustrates the clinical phenotype of a novel, likely pathogenic mutation in the ATP1A2 gene and can act as a useful benchmark for future cases in whom the same mutation is identified. This new mutation enhances our current knowledge of known mutations in the ATP1A2 gene.

\section{Acknowledgements}

None.

\section{Conflicts of Interest}

None.

\section{Funding}

None. 


\section{Ethical Approval}

None.

\section{Consent for Publication}

Written informed consent has been obtained for publication of this case presentation.

\section{Author Contribution}

AAM identified the patient and obtained consent. CJB wrote the first draft. AAM and CJB finalised later drafts together.

\section{References}

1. Russell MB, Ducros A (2011) Sporadic and familial hemiplegic migraine: Pathophysiological mechanisms, clinical characteristics, diagnosis, and management. Lancet Neurol 10: 457-470.

2. (2013) Headache Classification Committee of the International Headache Society (IHS). The International Classification of Headache Disorders. ( $3^{\text {rd }}$ edn), Cephalalgia 33: 629-808.

3. Thomsen LL, Eriksen EM, Romer SF, Andersen I, Ostergaard E, et al. (2002) An epidemiological survey of hemiplegic migraine. Cephalalgia 22: 361-375.

4. Malpas TJ, Riant F, Tournier-Lasserve E, Vahedi K, Neville BG (2010) Sporadic hemiplegic migraine and delayed cerebral oedema after minor head trauma: a novel de novo CACNA1A gene mutation. Dev Med Child Neurol 52: 103-104.

5. Spiller S, Friedrich T (2014) Functional analysis of human $\mathrm{Na}^{+} / \mathrm{K}^{+}$-ATPase familial or sporadic hemiplegic migraine mutations expressed in Xenopus oocytes. World $\mathrm{J}$ Biol Chem 5: 240-253. 